Portland State University

PDXScholar

8-7-2017

\title{
Developmental Trauma Disorder \& Effective Therapeutic Interventions
}

Ellena C. Rosenthal

Portland State University

Follow this and additional works at: https://pdxscholar.library.pdx.edu/honorstheses Let us know how access to this document benefits you.

\section{Recommended Citation}

Rosenthal, Ellena C., "Developmental Trauma Disorder \& Effective Therapeutic Interventions" (2017). University Honors Theses. Paper 472.

https://doi.org/10.15760/honors.472

This Thesis is brought to you for free and open access. It has been accepted for inclusion in University Honors Theses by an authorized administrator of PDXScholar. Please contact us if we can make this document more accessible: pdxscholar@pdx.edu. 
Developmental Trauma Disorder \& Effective Therapeutic Interventions

$$
\text { By Ellena Rosenthal }
$$

An undergraduate honors thesis submitted in partial fulfillment

of the requirements for the degree of

$$
\begin{gathered}
\text { Bachelor of Science } \\
\text { in } \\
\text { University Honors } \\
\text { and } \\
\text { Psychology }
\end{gathered}
$$

Thesis Adviser

Dave Fischer, Psy.D.

Portland State University

2017 
Table of Contents

Author's note

Abstract.

Introduction. .5

Literature Review....

Developmental Psychopathology....................... 9

Complex PTSD in Children and Adolescents...............10

Overview of DSM, Evaluation, and rejection of DTD .......13

Disorders of Extreme Stress Not Otherwise Specified.......17

Proposed Criteria for Developmental Trauma Disorder.......18

Understanding Developmental Trauma Disorder.............21

Effective Therapeutic Practices........................23

Component-Based Psychotherapy

Relational Treatment Framework

Risking Connection Attachment-Based Healing

Phase-Oriented Treatment

Yoga

Conclusion.................................................29

References.................................................... 32 
Everything within the pages of this literature review exists because of the tireless work of researchers, therapists and advocates who have dedicated their time to understanding and treating complex and developmental trauma and the detrimental effects it has in lives, in communities, and in the world. Thank you all for putting in the work, and thank you for continuing to write, produce, research and guide us towards a more compassionate and trauma-informed world.

I want to thank my Professor and advisor, Dr. Dave Fischer, who showed me the different realms and ways in which we can think and begin to comprehend the human condition. You introduced your students to a myriad of ways to understand and treat the psyche. That introduction fuels my scholarship in the field and the current focus of my life.

Lastly, though most importantly, I want to acknowledge the thousands of children, teenagers, and adults who have dealt with trauma. 


\begin{abstract}
In 2005, the Complex Trauma Taskforce, part of the National Child Traumatic Stress Network (NCTSN), constructed diagnostic criteria for a new diagnosis: Developmental Trauma Disorder (DTD). DTD serves as a more precise, accurate, and specific diagnosis for children and adolescents who have experienced multiple exposures to one or more developmentally adverse interpersonal traumas, which affect multiple areas of functioning and cause extreme interruptions in developmental processes throughout life (van der Kolk, 2005). A diagnosis of DTD describes the significant impairments and distress that characterize individuals living with developmental trauma, and provides clinicians a foundation and framework for working with therapeutic modalities specific to their trauma-informed clients' needs (D'Andrea, Ford, Stolbach, Spinazzola, \& van der Kolk, 2012). Although the construction of DTD is founded on clinical observations, empirical data, and more than twenty years of literature and research on developmental psychopathology, the American Psychiatric Association (APA) declined to include the DTD diagnosis in its most recent version of the Diagnostic and Statistical Manual of Mental Disorders (DSM-5) published in 2013 (van der Kolk, 2014). This literature review intends to provide background information on DTD, explain the importance of having the DTD diagnosis included in the DSM and the implications of the diagnosis' absence, and present effective therapeutic practices for developmental trauma.
\end{abstract}




\section{Introduction}

Childhood trauma is the United States' biggest public health concern (van der Kolk, 2005). Because of widespread, multifaceted, and often hidden causes of trauma that occur behind closed doors, maybe it should be labeled the nation's biggest silent epidemic (D'Andrea et al., 2012). In 2015, Child Protective Services, an entity within the Department of Health and Human Services, reported receiving 4 million referrals alleging maltreatment of 7.2 million children. $18 \%$ of the 7.2 million children were found to be victims of abuse or neglect, and, as in previous years, neglect was the most common form of child abuse ("Child Maltreatment 2015: Summary of Key Findings," 2017). The number of children and adolescents exposed to abuse and neglect, synonymous with trauma and traumatic stress, is more common than expected and continues to be under reported (van der Kolk, 2005). While the amount of trauma experienced by these individuals may be be underreported, the trauma endured by these same individuals is easier to enumerate.

The effects of traumatization and victimization follow children into their adult lives, and the cost of that childhood trauma is high (D'Andrea et al., 2012). According to Fang, Brown, Florence, \& Mercy (2012), 124 billion dollars is spent in one fiscal year on mental health and social services. Much of the expense attached to this trauma results from the pervasive and multifaceted symptomatology of children and adolescents exposed to multiple and/or chronic traumas during formative developmental years, including: developmental delays, somatic ills, impulse control issues, mood disorders, to participating in destructive and violent behaviors (van der Kolk, Pynoos, Cicchetti, Cloitre, D’Andrea, Ford \& Stolbach, 2009). Trauma does not only play out in the way that victims react to input, but also how they can perceive what is happening to them. Van der Kolk (2005) explores how exposure to multiple traumas or chronic and 
prolonged traumas have developmentally adverse effects on the mind and body and D'Andrea et al. (2012) explore how chronic maltreatment and repeated traumatization have detrimental neurological effects on development. With mind, body and development all affected negatively by trauma, it is difficult for the traumatized person to integrate sensory information, emotional information and cognitive information into one cohesive whole (van der Kolk, 2005). The capacity to form one's experiences into a cohesive whole that constitutes an identity and a foundation to find one's place in society is paramount to the development of secure attachments and sense of self, effective and positive internal working models, emotional and behavioral regulation, accurate reading of safety and environment, problem solving skills, and accurate categorizing of experience (van der Kolk, 2005). Given that tramatized individuals potentially have difficulty with one or more of these develpments, the cost of child trauma is indeed high.

In the 1990s, Kaiser Permanente and the Centers for Disease Control jointly funded a research study called the Adverse Childhood Experiences (ACE) study, which documented, among many things, that childhood trauma is common, with a recognition that occurrences are only increasing. The ACE study also confirmed connections between early childhood experiences and "depression, suicide attempts, alcoholism, drug abuse, sexual promiscuity, domestic violence, cigarette smoking, obesity, physical inactivity, and sexually transmitted diseases" (van der Kolk, 2005, p.3). So, not only are the tramatized struggling as young children, but their struggling does not stop as young people. In fact, the trauma continues to have negative effects all the way into adulthood since strong positive connections have been made between adverse childhood experiences and chronic health conditions experienced later in life, such as heart disease, cancer, diabetes, and liver diseases (van der Kolk, 2005). While the ACE study was significant, there were doubters. The doubt, however, was put to rest by the NCTSN 
longitudinal child trauma database and the thousands of studies looking at childhood victimization. Each of the studies showed similar, negative results: that chronic maltreatment and/or repeated traumatization experienced in early life significantly interfered with an individual's capacity to regulate internal states, wreaked havoc on the Central Nervous System, and interfered with important developmental functions (D'Andrea et al., 2012). Despite the presence of empirical support and vetted, no recognized diagnosis in the DSM labels the experiences, symptoms and co-occurring sequelae that are at the root of developmental trauma (van der Kolk, 2014).

Because of the lack of diagnostic criteria for childhood trauma included in the DSM, $40 \%$ of children affected by developmental trauma are commonly and incorrectly diagnosed with at least one other mood disorder, including Attention-Deficit/ Hyperactivity Disorder (ADHD), Post-traumatic Stress Disorder (PTSD), and Bipolar Disorder (D’Andrea et al., 2012). These incorrect diagnoses are a poor response to treatment frameworks and therapeutic interventions that are not trauma-informed or trauma based. For instance, a diagnosis of PTSD often infers that the individual experienced a circumscribed, isolated traumatic event (D'Andrea et al., 2012), rather than the repeated victimization many endure. The altered consciousness, amnesia, hypermnesia, dissociation, depersonalization, derealization, flashbacks, nightmares, attention regulation, orientation in time and space, and issues with sensorimotor development as results of trauma cannot be accurately described by a PTSD diagnosis (D'Andrea et al., 2012), nor can they be medicated by a prescription for an ADHD symptom. With correct framework for naming the trauma, and incorrect therapeutic attempts to help individuals heal, there needs to be a better method of diagnosis and treatment. 
In an effort to address the misdiagnoses of trauma survivors, comorbid conditions are often employed to describe the vast spectrum of symptoms related to trauma, but the diagnosis of comorbid disorders for individuals affected by developmental trauma do not aid in the recovery or healing process. Trying to apply incorrect diagnoses and therapies inappropriately means variables get lost in translation and applied treatment approaches and frameworks are not effective (van der Kolk et al., 2009). Without a change to a framework that centers on the trauma that shapes trauma informed individuals, future research and understanding of developmental trauma may become distorted and inaccurate. More significantly, individuals will continue to suffer from preventable distress. Given the right interventions and tools at the outset, these individuals might start to live more manageable lives (van der Kolk et al., 2009) with less recurring trauma and suffering.

However, in the past thirty years, a lens through which to treat and understand developmental and complex trauma has emerged. DTD approaches trauma as an expression of vast internal disorganization centered on developmental trauma as the core source of deficiencies and/or abnormalities in multimodal functioning of brain and body, even though DTD was rejected for inclusion in the most recent (2013) published version of the DSM-5 (van der Kolk, 2014).

Although the disorder has yet to be recognized by the American Psychiatric Association, mental health practitioners, researchers, and providers in the field of traumatic stress recognize, affirm, and treat those suffering from DTD. Literature suggests a recognized diagnosis would improve current trauma-informed interventions, guide development of newer treatments, create a more precise understanding of who may be affected by developmental trauma, allow for 
insurance reimbursement, and aid research and scientific study, centering the effects of developmental trauma along with lived experience (D'Andrea et al., 2012).

A recognized diagnosis of DTD would reduce diagnostic confusion and could reduce the pathologizing many trauma survivors have internalized over years of mistreatment and misdiagnosis (D’Andrea et al., 2012).

\section{Developmental Psychopathology}

The Post-traumatic Stress Disorder classification system in the 1980s led to extensive scientific studies of diagnoses in the field of traumatic stress (van der Kolk et al., 2009). From the 1980s until now, developmental psychopathology within the field of traumatic stress, paying special attention to children and adolescents, has documented the effects of interpersonal traumas and disruptions in caregiving systems and the effect they have on an individual's development of affect regulation, attention, cognition, perception, and interpersonal relationships. Developmental psychopathology can be best understood as a framework to examine clinical diagnoses often seen among child and adolescent populations (van der Kolk et al., 2009). Even without a DTD diagnosis, a trauma-centered framework considers contextual factors, categorical and dimensional models, risk and resilience, and developmental pathways when examining these clinical diagnoses (Drabick \& Kendall, 2010). Looking at DTD as the diagnosis, rather than trying to force individuals into other mis-fitting diagnoses, the emergence and maintenance of comorbid/co-occurring diagnoses in children and adolescents is understood as interactions among biological, psychological and social factors (Drabick et al., 2010) in a developmental psychopathology framework. To be most effective at understanding how trauma affects individuals at micro, meso, and macro levels, interactions among biological, psychological and 
social factors must be emphasized. Developmental psychopathology supports the understanding of trauma and traumatic stress by documenting the effects of adverse childhood experiences in early life, and the disruption these experiences may have on brain development, neuroendocrinology, and immunology (van der Kolk et al., 2009). By showing that these traumas do not affect one part of the body, one part of development or have one solution, developmental psychopathology helps form greater understanding of therapies to be applied or diagnoses to consider when a traumatized individual is being treated.

\section{Complex-Post Traumatic Stress Disorder in Children and Adolescents}

Research conducted within the scope of developmental psychopathology repeatedly documents that children and adults who are exposed to childhood trauma show that trauma does not occurr in isolation. Instead, these presentations best fit diagnoses of co-occurring, chronic types of victimization, or Complex-Post Traumatic Stress (c-PTSD) (van der Kolk et al., 2009).

Victimization is best understood when addressing interpersonal traumas seen in children as:

"...harm that comes to individuals because other human actors have behaved in ways that violate social norms. Even though we sometimes refer to people as "victims of hurricanes",... or accident victims." The more common reference for the term victimization is interpersonal victimization. In interpersonal victimization, the elements of malevolence, betrayal, injustice, and immorality are more likely to be factors than in accidents, disease, and natural disasters (Finkelhor, 2008, p.188).

In children, specific types of trauma have the most devastating effects: acts of

commision, such as sexual assault, and acts of omission, such as neglect or abandonment. In acts of omission, withdrawal or lack of resources has the probability of threatening the child's very survival and physical well being, especially occurring at the hands of caretakers (Cloitre, Stolbach, Herman, van der Kolk, Pynoos, Wang, \& Petkova, 2009). The understanding of cPTSD became more widespread when a book, published in 1992, provided a new way of aiding, 
treating, and understanding trauma survivors. Trauma and Recovery: The Aftermath of Violence

- From Domestic Abuse to Political Terror presents a concept of understanding trauma that was not through the lens of the traditional diagnosis of PTSD, which was often based off of circumscribed events, and usually occurs as the aftermath of a one-time traumatic event (Herman, 1992).

"They [survivors of PTSD] are based on the prototypes of combat, disaster, and rape. In survivors of prolonged, repeated trauma, the symptom picture is often more complex. Survivors of prolonged abuse develop characteristic personality changes, including deformations of relatedness and identity. Survivors of abuse in childhood develop similar problems with relationships and identity; in addition, they are particularly vulnerable to repeated harm, both self inflicted and at the hands of others. (Herman,1992, p.119)

As a better understanding of the multiple layers of trauma an individual endures became

part of the understood cannon, the types of of co-occurring, chronic types of victimization and

traumas could also be shown via alterations in multiple categories:

Alterations in affect regulation, like persistent dysphoria, chronic suicidal preoccupation, selfinjury, explosive or extremely inhibited anger, compulsive or extremely inhibited sexuality (Herman, 1992)

Alterations in consciousness, like amnesia or hypermnesia for traumatic events, transient dissociative episodes, depersonalization/derealization, reliving experiences, either in the form of intrusive post-traumatic stress disorder symptoms or in the form of ruminative preoccupation (Herman, 1992)

Alterations in self-perception, like sense of helplessness or paralysis of initiative, shame,guilt, and self-blame, sense of defilement or stigma, sense of complete difference from others, which may include sense of specialness, utter aloneness, belief no other person can understand, or nonhuman identify (Herman, 1992)

Alterations in perception of perpetrator, like preoccupation with relationship with perpetrator, includes preoccupation with revenge, unrealistic attribution of total power to perpetrator, idealization or paradoxical gratitude, sense of special or supernatural relationship, acceptance of belief system or rationalizations of perpetrator (Herman, 1992)

Alterations in relations with others, like isolation and withdrawal, disruption in intimate relationships, repeated search for rescuer, persistent distrust, repeated failures of self protection (Herman, 1992)

Alterations in systems of meaning, like loss of sustaining faith, sense of hopelessness and despair (Herman, 1992) 
As the alterations of trauma presentation became better understood, clinicians proposed that the DSM diagnostic system and accompanying criteria were inadequate for the treatment of extreme trauma survivors. They added that the preliminary treatment for the effects of trauma were often lost in clinical settings because the symptom set an individual disclosed might not be connected - for ambiguous reasons - to past traumatic experience(s), often in early childhood. Clinicians treating survivors of trauma, and those impacted, continued to see clients who had experienced chronic and/or prolonged trauma but showed many additional symptoms alongside and separate from the formal symptomatology of PTSD ("Complex PTSD," 2016). Trauma permitted researchers and clinicians to start to understand that trauma would not be a cause and effect relationship, but one that would need new approaches and understanding of long-standing trauma.

Some in the field of developmental psychopathology felt that the psychiatric field understands trauma through a singular, often linear, lens which results in fragmented approaches to treatment that are often ineffective for trauma survivors. Patients continue to be assigned comorbid diagnoses, none of which are trauma-related or trauma-treated. The proposed disorder of c-PTSD would recognize the impact of prolonged and chronic traumas and help facilitate development of effective forms of treatment (Herman, 1992). Naming c-PTSD would be an essential step in recognizing the immense turmoil individuals endure due to trauma, and would begin to shape the field of traumatic-stress studies in a new, multi-modal way (Herman, 1992). Large populations of people, specifically children and adolescents who also experienced the sequelae of chronic and prolonged trauma, would be recognized and effectively treated with a cPTSD diagnosis, an important step toward developing a DTD diagnosis.

"It is an attempt to find a language that is at once faithful to the traditions of accurate psychological observation and to the moral demands of traumatized people. It is an 
attempt to learn from survivors, who understand more profoundly than any investigator the effects of captivity." (Herman, 1992, p.122)

\section{Overview of DSM, Evaluation, and rejection of DTD}

\section{Overview of DSM and Evaluation}

In 1921, an entity called the American Medico-Psychological Association - now referred to as the American Psychological Association - joined with the New York Academy of Medicine and created what is know known as the first nationally recognized and acceptable psychiatric classification system. This first version was designed for diagnosing patients with severe psychiatric and neurological ailments.

After World War II, it was clear that there was a need for a broader classification system that encompassed outpatient presentations of people who had endured severe trauma by serving in war. The World Health Organization (WHO) published the $6^{\text {th }}$ edition of the International Classification of Disease, which included 10 categories for psychosis and psychoneuroses and seven categories of disorders of character, behavior, and intelligence. The first edition of the DSM was published in 1952 by the APA, and was the first official manual of mental disorders focused on use for clinical settings ("DSM History," 2017). The DSM was developed as an evidence-based manual used to characterize symptoms and behaviors of groups of people. WHO had oversight of the first DSM because the organization felt that this version contained issues with diagnoses, and provided the APA with recommendations to better the DSM-II. These recommendations were not applied to the DSM-II. The first psychiatrist-in-chief at John Hopkins Hospital introduced the word "reaction" into the first version of the DSM to explain to clinicians the psychological view that "mental disorders represented reactions of the personality to psychological, social, and biological factors" (van der Kolk, 2014). The revised DSM-II eliminated "reaction," which was its only change (“DSM History," 2017). 
Often referred to as the "Bible of Psychiatry," the DSM is based on collective clinical and scientific knowledge and lists hundreds of mental disorders within the manual ("DSM History," 2017). As diagnoses have changed, there have been several versions of the DSM with multiple revisions. The first edition of the DSM-III in the 1980s was an imprecise diagnostic system, and the APA made an official announcement that it should not be used for forensic or insurance purposes (van der Kolk, 2014). The current edition - the DSM-5-was researched and prepared for a decade before its publication in 2013. Preparation for the $5^{\text {th }}$ edition meant that evaluations of current diagnostic systems took place employing different task groups, "white papers" were submitted, and 13 scientific conference groups supported by the National Institute of Health (NIH) provided oversight and review of the $5^{\text {th }}$ edition ("DSM History," 2017). The DSM has tried to keep up with new understandings of trauma, mental health and treatment, but with each version needing at least a decade to be produced and reviewed, one hopes that the next version may contain the DTD diagnosis.

\section{Rejection of DTD by DSM}

Dr. Bessel van der Kolk, an internationally recognized leader in the study and treatment of trauma, was one of four experts in the field of traumatic stress to first propose the diagnosis of DTD be added to a revised version of the DSM-III. Van der Kolk and others, all of whom later created the Complex Trauma Taskforce, were told by the APA to conduct a field trial. This field trial compared the problems of different groups of traumatized individuals in order to show the need for a developmentally appropriate trauma diagnosis. The study interviewed 525 adult patients and looked to see if particular populations of these adult patients suffered from different “constellations" of problems (van der Kolk, 2014). 
Results yielded three different groups within the 525: people with histories of childhood physical and/or sexual abuse by caregivers, recent victims of domestic violence, and individuals who had just been through a natural disaster. The largest discrepancy occurs between people who were victims of childhood physical and/or sexual abuse, and people who had survived natural disasters. Adults who were self-described victims of physical and sexual child abuse showed issues with concentration, problems with negotiating intimate relationships, had large gaps in memory consolidation, engaged in self-destructive behaviors and had multiple medical problems. This symptom set was rare in the group of adults who had recently survived natural disasters (van der Kolk, 2014). The findings showed clear trends linked to specific events and specific outcomes in the lives of the traumatized individuals.

Dr. van der Kolk presented the field trial findings to the PTSD workgroup in charge of suggesting revisions to the new edition of the DSM. The work group voted to add a new diagnosis for victims of interpersonal trauma labeled Disorders of Extreme Stress Not Otherwise Specified and/or Complex PTSD. In 1994, the diagnoses were not included in the revised edition of the DSM. This rejection was discouraging - if the condition does not exist, one cannot develop a treatment for said trauma, and some felt that organizations and individuals responsible for shaping the diagnostic system failed to recognize the evidence of the effects of developmental and complex trauma (van der Kolk, 2014).

After the initial rejection in the 1990s, a taskforce made up of 12 clinicians and researchers specializing in childhood trauma convened twice a year for four years to draft a proposal for DTD. This proposal took from 130 different studies that reported on 100,000 abused and neglected children and adolescents. In February of 2009 the proposed diagnosis of DTD was submitted to the APA - a single diagnosis of developmental trauma as opposed to multiple 
diagnoses which failed to indicate the locus of the issue (van der Kolk, 2014). Organizations like the National Association of State Mental Health Program Directors requested that the APA include developmental trauma to its list of priority areas.

Months after the proposed diagnosis of DTD was submitted to the APA, the chair of the relevant DSM subcommittee announced that DTD was unlikely to be included in the next edition of the DSM, the DSM-5, one of the reasons being that the proposed diagnosis did not provide enough evidence for "this niche diagnosis," and further stated that "the notion that early childhood adverse experiences lead to substantial developmental disruptions is more clinical intuition than a research based fact... [the] statement is commonly made but cannot be backed up by prospective studies." (van der Kolk, 2014).

Additional feedback from the APA about the rejected diagnosis focused on the need to make sure research was accurate and showing no weakness in understanding who and how to correctly diagnose people with DTD. This would secure that the diagnosis would not be misused or overused ("What A New Diagnosis Would Include," 2007). The DSM-5 subcommittee also explained that the Complex Trauma Taskforce had provided too much data on DTD and not the right kind of data. The APA subcommittee wanted to see research on how and if trauma is related to possible genetic transmission, environmental risk factors, temperamental antecedents, bio-markers, familial patterns, and treatment responses to trauma in order for future inclusion within the DSM (Wylie, 2013). Since DTD is still considered a proposed diagnosis, the research needed for future inclusion cannot be conducted since funding - through the APA - is not available for proposed diagnoses (Wylie, 2013).

\section{Disorders of Extreme Stress Not Otherwise Specified}


During the six years prior to the DSM-IV's release in 1994, the American Psychiatric Association used field trials to investigate and analyze what, if any, impacts to the PTSD diagnosis would occur with proposed changes - like looking at the psychopathology of chronic developmental trauma (Sar, 2011). In conjunction with Dr. Herman and the DSM-IV field trial committee, a trial was conducted referred to as Disorders of Extreme Stress Not Otherwise Specified (DESNOS), which showed the differential impact of interpersonal trauma on adults who as children were exposed to chronic interpersonal traumas, compared to patients who as mature adults were exposed to assaults, disasters, or accidents (van der Kolk et al., 2009). DESNOS field trial showed that out of the population researched, $92 \%$ of individuals met the criteria for a traditional PTSD diagnosis, along with a c-PTSD and DESNOS diagnosis (“Complex PTSD,” 2016).

This field trial resulted in the diagnostic category DESNOS, and a DESNOS symptom set was incorporated to the already formulated, traditional diagnosis of PTSD, listed under “associated and descriptive features of PTSD" in the 4th edition of the DSM (American Psychiatric Association, 1994). The result was not a free-standing diagnosis like c-PTSD, which conceptualizes trauma in a multidimensional way, centering subpopulations like children and adolescents which many researchers and clinicians focusing on developmental psychopathology had hoped it would do. Instead, literature suggests that because of the atheoretical character of the DSM-IV and overlapping diagnostic categories leading to comorbidity issues, DESNOS failed to stand alone as its own diagnostic category (Sar, 2011).

The differences between adult onset PTSD, c-PTSD and the effects of interpersonal violence on children, along with the need to research and treat these children with effective 
strategies were some reasons for the creation of the National Child Traumatic Stress Network in 2001 (van der Kolk et al., 2009).

NCTSN's mission is to raise the standard of care and to improve access to services for traumatized children, their families, and communities. The organization extends traumainformed care, practices, and resources to all types of child-serving systems, and has funded many studies in the realm of child welfare, trauma, and recommendations for improvement in treatment and child servic-e agencies. NCTSN as an organization became aware, as patients continued to be first diagnosed, and then treated, that a different trauma-informed diagnosis was needed, one that captured the lived realities in the totality of their experiences. A database of statistical information was created on almost twenty thousands children who had been treated at various sites within the network, and a collection of research articles about the effects of childhood abuse and neglect concurred (van der Kolk, 2014).

In 2005, the NCTSN conducted a survey of 1,699 children receiving trauma-focused treatment across 25 affiliated sites in the United States. This survey showed that $78 \%$ of the children had been exposed to multiple and/or prolonged interpersonal traumas, and less than $1 / 4$ of the 1,699 children met diagnostic criteria for PTSD (van der Kolk et al., 2009).

Two years prior, NCTSN Complex Trauma Taskforce conducted a clinician survey on trauma exposure for children who were receiving assessment and/or intervention services. It was found that $78 \%$ of the children had experienced more than one trauma type, and that the initial exposure to trauma was age 5 (van der Kolk et al., 2009).

In 2009, Dr. van der Kolk proposed the creation of the DTD, and the NCTSN devised the proposed diagnosis criteria for DTD with the hope that it would be included in the next version of the DSM (van der Kolk et al., 2009). Before this proposal, most literature and research 
methods looking at traumatic stress focused on single types of trauma and stand- alone symptom sets, warranting isolated diagnosis or comorbid diagnoses (Denton, Frogley, Jackson, John, \& Querstret, 2017).

The creation of DTD described the most clinically significant symptoms seen throughout years of research and care, and described broad domains of impairment and distress which occur within the DTD symptom set, and not within the only trauma-specific diagnosis of PTSD. DTD symptoms and impairments are functionally different than normal behavioral responses to stressors or changes in a child or adolescent's psychobiodevelopment. Typical forms of chronic traumatization, leading to DTD, could be from events such as child abuse: sexual, emotional, or physical, or from neglect: emotional, or physical. At last, the principle that trauma had long lasting effects and created a different circumstances was recognized as different from an individual who had noy been tramatized and had their life view attached to the trauma and its effects.

The proposed DTD criteria helped narrow down who has developmental trauma, aid in the creation and continued practice of effective forms of treatment, and further validates the diagnosis in the field of psychology and traumatic stress (van der Kolk et al., 2009). Though the proposed diagnostic criteria and disorder were backed by years of empirical evidence, statistical findings, interviews, survey results, and lived experiences of those affected by developmental trauma, the APA declined to include DTD in its most recent version of the DSM (van der Kolk et al., 2009).

\section{CONSENSUS PROPOSED CRITERIA FOR DEVELOPMENTAL TRAUMA DISORDER}

A. Exposure. The child or adolescent has experienced or witnessed multiple or prolonged adverse events over a period of at least one year beginning in childhood or early adolescence, including: 
A. 1. Direct experience or witnessing of repeated and severe episodes of interpersonal violence; and

A. 2. Significant disruptions of protective caregiving as the result of repeated changes in primary caregiver; repeated separation from the primary caregiver; or exposure to severe and persistent emotional abuse

B. Affective and Physiological Dysregulation. The child exhibits impaired normative developmental competencies related to arousal regulation, including at least two of the following:

B. 1. Inability to modulate, tolerate, or recover from extreme affect states (e.g., fear, anger, shame), including prolonged and extreme tantrums, or immobilization

B. 2. Disturbances in regulation in bodily functions (e.g. persistent disturbances in sleeping, eating, and elimination; over-reactivity or under-reactivity to touch and sounds; disorganization during routine transitions)

B. 3. Diminished awareness/dissociation of sensations, emotions and bodily states

B. 4. Impaired capacity to describe emotions or bodily states

C. Attentional and Behavioral Dysregulation: The child exhibits impaired normative developmental competencies related to sustained attention, learning, or coping with stress, including at least three of the following:

C. 1. Preoccupation with threat, or impaired capacity to perceive threat, including misreading of safety and danger cues

C. 2. Impaired capacity for self-protection, including extreme risk-taking or thrill-seeking

C. 3. Maladaptive attempts at self-soothing (e.g., rocking and other rhythmical movements, compulsive masturbation)

C. 4. Habitual (intentional or automatic) or reactive self-harm

C. 5. Inability to initiate or sustain goal-directed behavior

D. Self and Relational Dysregulation. The child exhibits impaired normative developmental competencies in their sense of personal identity and involvement in relationships, including at least three of the following:

D. 1. Intense preoccupation with safety of the caregiver or other loved ones (including precocious caregiving) or difficulty tolerating reunion with them after separation

D. 2. Persistent negative sense of self, including self-loathing, helplessness, worthlessness, ineffectiveness, or defectiveness

D. 3. Extreme and persistent distrust, defiance or lack of reciprocal behavior in close relationships with adults or peers

D. 4. Reactive physical or verbal aggression toward peers, caregivers, or other adults

D. 5. Inappropriate (excessive or promiscuous) attempts to get intimate contact (including but not limited to sexual or physical intimacy) or excessive reliance on peers or adults for safety and reassurance 
D. 6. Impaired capacity to regulate empathic arousal as evidenced by lack of empathy for, or intolerance of, expressions of distress of others, or excessive responsiveness to the distress of others

E. Posttraumatic Spectrum Symptoms. The child exhibits at least one symptom in at least two of the three PTSD symptom clusters B, C, \& D.

F. Duration of disturbance (symptoms in DTD Criteria B, C, D, and E) at least 6 months.

G. Functional Impairment. The disturbance causes clinically significant distress or impairment in at two of the following areas of functioning:

- Scholastic: under-performance, non-attendance, disciplinary problems, drop-out, failure to complete degree/credential(s), conflict with school personnel, learning disabilities or intellectual impairment that cannot be accounted for by neurological or other factors.

- Familial: conflict, avoidance/passivity, running away, detachment and surrogate replacements, attempts to physically or emotionally hurt family members, non-fulfillment of responsibilities within the family.

- Peer Group: isolation, deviant affiliations, persistent physical or emotional conflict, avoidance/passivity, involvement in violence or unsafe acts, age- inappropriate affiliations or style of interaction.

- Legal: arrests/recidivism, detention, convictions, incarceration, violation of probation or other court orders, increasingly severe offenses, crimes against other persons, disregard or contempt for the law or for conventional moral standards.

- Health: physical illness or problems that cannot be fully accounted for physical injury or degeneration, involving the digestive, neurological (including conversion symptoms and analgesia), sexual, immune, cardiopulmonary, proprioceptive, or sensory systems, or severe headaches (including migraine) or chronic pain or fatigue.

- Vocational (for youth involved in, seeking or referred for employment, volunteer work or job training): disinterest in work/vocation, inability to get or keep jobs, persistent conflict with co-workers or supervisors, under-employment in relation to abilities, failure to achieve expectable advancements. (van der Kolk, 2009, p. 5).

\section{Understanding Developmental Trauma Disorder}

All children learn to regulate their physical and emotional behaviors by anticipating their caregivers' responses, dependent on the type of attachment the child has to the caregiver(s). Children learn and construct internal working models based off of affective and cognitive characteristics shown in primary early relationships. These early experiences occur in the context of brain development, neural development, social development, and social interactions, all of 
which are interconnected with internal working models, and early attachment patterns (Moretti et al., 2004).

The processes and systems above inform how the child and/or adolescent processes, stores, categorizes and understands information throughout their life (Schimmenti \& Caretti, 2016). There is no developmentally appropriate diagnosis in the DSM for children and adolescents who deal with the devastating effects of trauma and its sequelae. The current diagnostic criteria of PTSD and other sub-symptom sets do not fully capture, explain, or begin to provide effective treatment modalities for the effects of severe physical harm of prolonged, repeated, trauma on an individual's mind, body, and spirit, especially during developmental years (van der Kolk, 2013). In clinical settings traumatized children and adolescents are usually diagnosed with a variety of psychiatric labels, but none of the labels explain the profound developmental disturbances seen in cases of victimization (van der Kolk, 2002). Unless correctly labeled as developmental trauma, children exhibiting specific behaviors may be labeled as oppositional, rebellious, unmotivated, and/or antisocial by school and/or correctional entities (van der Kolk, 2009).

Using NCTSN's core data set, 658 adolescents reported involvement in the juvenile justice system and in 2013 the NCTSN reported that $90 \%$ of those justice-involved youth had experience with some type of traumatic event (Moretti \& Peled, 2004). 70\% of the 658 cohort meet criteria for a mental health disorder, with only $30 \%$ of that same youth sample meeting the diagnostic criteria for PTSD as laid out in the DSM-IV. Unfortunately there is little evidence that children and adolescents who have experienced early onset trauma outgrow the problems that occur. People with early histories of abuse and neglect are found to suffer into their adult lives, often reoffending and continuing to have pervasive psychiatric problem (van der Kolk, 2002). 
The current diagnostic system does not account for Developmental Trauma. Diagnosing children and adolescents with comorbid disorders, including PTSD, and not viewing their experience through the lens of developmental psychopathology and complex traumatic stress continues to perpetuate the victimization and dehumanization most of these individuals have already experienced in their life. The further pathologizing of experiences can deter people from getting the treatment that they may need to thrive. A diagnosis of DTD will help organize and treat the incredibly complex emotional, behavioral, and neurobiological sequelae of childhood trauma that continues to be misdiagnosed and at times ignored by the field of psychiatrics (D’Andrea et al., 2012).

\section{Effective Therapeutic Practices for DTD}

There is no one-size-fits-all approach when it comes to trauma-informed therapy and trauma treatment, especially for adult survivors of developmental trauma (Grossman, Spinazzola, Zucker, \& Hopper, 2017). Adult clients who have lived through developmental trauma may have treatment-resistant psychological and psychiatric conditions, therefore may need to try different forms of trauma treatment.

\section{Component-Based Psychotherapy}

The Trauma Center at Justice Resource Institute has come up with therapies for adult survivors of childhood maltreat. It is noted that these type of trauma survivors "tend to show greater impairment in the capacity to establish and maintain safe, healthy, and loving relationships; to process more negative self-image, worth, or esteem; to be more likely to internalize their distress, leading to more frequent difficulties with depression, anxiety, social withdrawal, and isolation; and to engage in more maladaptive forms of coping, including greater 
prevalence of self-injury, alcohol and substance abuse, and other risk-taking behaviors including sexual acting out" (Grossman et al., 2017, p.87).

CBP is used at The Trauma Center and is "an evidenced-informed model that bridges, synthesizes, and expands upon several existing schools, or theories, of treatment for adult survivors of traumatic stress" (p.87). Some of the theories are more traditional ones, like psychoanalysis, and others are more current approaches, like those informed by theories stemming from feminist thought (Grossman et al., 2017). Integration of four main components occur in therapy: relationship, regulation, working with dissociated aspects of the self, and narrative (p.88). Central to the four components is the relationship between therapist and client as being significantly important and primary for healing trauma and trauma-related issues. The client must feel that their therapist is attuned to their needs and that there is quality and stability within their relationship. This proves to determine if the client will positively benefit from therapy (p.89).

Adults who have experienced developmental trauma often times have problematic attachment styles, and may at first relate to the therapist in different ways, sometimes overly clingy or other times avoidant and angry. CBP is an effective therapeutic framework, especially for these types of clients, as it supports therapists being fully present, acutely attuned to their client, and allows for noticing “the relational ebbs and flows reflected in client's' body language, tone, eye contact, and so forth" (Grossman et al., 2017 p.89).

This, if done well, aids clients' regulation and often times fluctuating and intense emotional states that can be projected onto the therapist and intensify in the therapist-client relationship. It is important to note that with CBP, the therapist's presence in the room is one of the most important aspects of therapy (Grossman et al., 2017). 


\section{Relational Treatment Framework}

Relational Treatment Framework (RTF) is especially effective when treating individuals exposed to chronic and/or prolonged interpersonal violence, neglect, and abuse. When these things occurs at the hands of primary caregivers, these children, whom later become adults, oftentimes develop issues with maintaining healthy attachments within interpersonal relationships, have issues managing, relating and connecting with other people, and have a chasm in cultivating healthy relationships that offer security, stability, and safety (Pearlman \& Courtois, 2005).

These alterations within relationships occur in all facets of life. Often chronically victimized, neglected and abused people continue to experience retraumatization through relationships. For instance, individuals may form relationships with others who have experienced or are experiencing similar trauma(s) and/or loss, and who have similar dysfunctional behaviors and relational deficits as the individual. Often times past relationships and patterns of attachment are reenacted. The effects of reenacting attachment patterns or dysfunctional/unsafe relationships can cause more interpersonal damage to the individual, continue to retraumatize the individual and push them to either a hyperaroused or dissociative state(s), and adds to the instability, mistrust and grievances often felt towards others through the lens of those chronically traumatized (Saakvitne, Gamble, Pearlman, \& Lev, 2000).

Using RTF, issues with attachment and inner and outer modulation of behavior, affect and emotion may be healed within the context of a patient-therapist relationship. The development of a therapeutic relationship is characterized by four fundamentals: respect, information, connection, and hope (RICH) (Saakvitne et al., 2000). A safe, stable, recioprical and attuned therapist and therapeutic relationship can provide chronically traumatized clients an 
understanding of coping mechanisms and that the effects of trauma can be experienced, explored, shared, understood, verbally addressed, and ultimately resolved (Pearlman et al., 2005).

Stable and secure attachments are critically important for people to function productively with self and others, and a therapeutic alliance may be the first time chronically traumatized people feel a stable and secure attachment to someone, without disruption occurring within that relationship. A secure and safe relationship based on attachment models is essential for healing and continued identity development and formation not affected by the grasps of trauma. A fundamental of RTF is working with clients to understand their Inner Working Models (IWM) and how these models affect attachment styles and maladaptations experienced in life. In 1988 John Bowlby explained that exploring and understanding patients' Inner Working Models within the context of a therapeutic relationship can help the patient understand attachment and expectations they may have in relations with others and the therapist (Pearlman et al., 2005).

\section{Risking Connection Attachment-Based Healing}

Risking Connection is trauma-focused curriculum and training designed for people within mental health settings who work with survivors of childhood abuse (Pearlman et al., 2005). RC provides a model of attachment-based healing which can be applied within a relational therapeutic framework. $\mathrm{RC}$ takes four main approaches as effective goals within trauma-informed and trauma-specific therapy: (1) delineating psychological realms affected by traumatic experiences, (2) combining the relational and attachment perspectives, (3) providing relational guidance and goals for treatment, (4) emphasizing the importance of the treatment provider's experience in highlighting and integrating an understanding of countertransference and vicarious traumatization onto treatment (Pearlman et al., 2005). 
The model of attachment-based healing described in RC curriculum occurs when a therapist is effectively attuned to their client and is focused on relationship patterns within the therapeutic alliance, and is able to work and engage with relational repair and attachment disruption. People effected by chronic and/or prolonged trauma with insecure-preoccupied, insecure-dismissing, disorganized, disoriented, or dissociative attachment styles respond well to attachment-based healing, and may benefit from structured and organized interventions within the therapeutic relationship. This could look like a phase-oriented model of treatment or a sequenced model of treatment utilized by the therapist (Pearlman et al., 2005).

\section{Phase-Oriented Treatment}

Phase-oriented treatment is proven effective when working with traumatized individuals, though it is important to note that any treatment(s) involving traumatized individuals must be placed according to the degree of involuntary intrusion of trauma experienced by the person and how that person deals with the involuntary intrusion (van der Kolk, 2002). The therapist conducting phase-oriented treatment must not just respect but also understand how coping methods, though maladaptive and possibly destructive, have allowed the client to survive and acquire resilience with the memories and blueprint of trauma.

A phase-oriented treatment model applied in a therapeutic relationship and/or clinical setting is as follows: (1) establishing diagnoses, including prioritizing the range of problems suffered by individual, (2) designing a realistic phase-oriented treatment plan, consisting of: 1 . Stabilization, including identification of feelings by verbalizing somatic states, 2. Deconditioning of traumatic memories and responses, 3. Integration of traumatic personal schemes, 4 . Reestablishing secure social connections and interpersonal efficacy, 5. Accumulating restitutive emotional experiences. With the above in mind, effective therapeutic practice of phase-oriented 
treatment deals with stabilization of the patient first and foremost, and may need to refocus many times on stabilization (van der Kolk, 2002).

\section{Yoga Practice}

Yoga and the connecting concept of mindfulness continues to be one of the most effective forms of treatment for traumatized individuals. Chronically traumatized individuals have disturbances with body regulation, emotion regulation and affect regulation, and will usually move between a state of hyperarousal and a state of dissociation. Trauma takes over a person's relationship to their body and the understanding of their body's needs, like sleep, pleasure, hunger, and exercise. The mindfulness that the practice of yoga fosters for individuals helps with increased experience of body, emotion regulation, and aiding the person in noticing different feelings in their body. Feelings like hunger, hurt, physical loneliness or fear-related sensations that counteract avoidance symptoms (Price, Spinazzola, Musicaro, Turner, Suvak, Emerson, \& van der Kolk, 2017). Individuals with a trauma diagnosis are often not aware of the connection between internal reactions and things occurring in the environment, and the practice of yoga can heal this rift.

Trauma-informed, time-limited yoga is effective for treating the symptom set associated with chronic trauma and traumatic stress, and lowers things like inflammatory hormones, stress levels, and adrenal levels (Price et al., 2017). Affect-attunement occurs in yoga practice which is often needed when treating traumatized patients. Yoga aids with understanding physical sensations and putting them all into a coherent whole. Learning to modulate breath is effective when traumatized patients are faced with a past or current trauma trigger (van der Kolk, 2002). 


\section{Conclusion}

In 2013 the Journal of Traumatic Stress published a research study that assessed, using archival data, whether the proposed diagnostic criteria of DTD adequately reflected the experiences and symptoms of traumatized urban youth (Stolbach, Mineshew, Rompala, Dominguez, Gazibara, \& Finke, 2013). Research looking at the proposed diagnosis of DTD lacks funding since its not yet listed in the DSM, which in turn affects moving forward with the diagnosis. Furthermore, things like screening mechanisms and standardized measures for DTD have yet to be developed so the study utilized diagnostic tools and psychometrics that were not intended to diagnosis DTD. The 2013 study used 214 children who had received services from an urban child trauma treatment center after experiencing one or more PTSD Criterion A stressors cited in the DSM-IV (Solbach et al., 2013).

The results indicated strong support for the DTD construct as a diagnostic category and proposes that tools that allow clinicians and researchers to study the effects of complex trauma in children is an important next step, one of them being that future studies should emphasize variables like age, gender, and cultural background to validate developmental trauma (Stolbach et al., 2013).

Expressions of complex trauma symptoms have been studied in Ugandan child soldiers, emphasizing the prevalence of DTD in this specific chronically traumatized population (Klasen, Oettingen, Daniels, Post, Hoyer, \& Adam, 2010). The article published in the Journal of Aggression, Maltreatment \& Trauma studied this population through the lens of a DTD diagnosis and a PTSD diagnosis, looking at things like the effects of war trauma and domestic violence. Results indicated that $80 \%$ of the sample of former child soldiers met criteria for DTD, whereas only $33 \%$ of the sample met criteria for PTSD (Klasen et al., 2010). Domestic and community 
violence were significantly related to DTD symptoms but not PTSD symptoms. Traumatic war experiences were significantly related to PTSD symptoms but not to DTD symptoms (Klasen et al., 2010).

Although DTD is a proposed diagnosis, clinicians and therapists of all sorts are still using developmental trauma as a way to assess and effectively treat patients, and as a way to inform therapeutic frameworks. Different organizations have goals to push towards a more precise diagnosis for developmental trauma, and places like The Trauma Center at Justice Resource Institute continue to publish trauma related research studies, and provide effective therapeutic interventions for therapists working with clients with developmental and complex trauma (Grossman et al., 2017).

There still remains a considerable push to have DTD recognized as an actual diagnosis in the pages of the DSM, though some still feel that the diagnostic criteria as it stands is ineffective and inaccurate, and articles continue to be published about the debate and contentiousness with including the diagnosis in the DSM (Schmid, Peterman, \& Fegert, 2013). Some literature states that the way DTD is explained in its current form conflicts with traditional diagnostic systems and may present a higher risk for therapists to miss the presentation of comorbid disorders in patients, in turn missing the opportunities for " effective (psycho-) pharmaceutical treatment" (Schmid, 2013, p.6). The debate of adding a formal DTD diagnosis into the DSM does not revolve around whether or not there is a correlation between traumatic experiences and related symptoms. Rather, it is about how to treat psychopathological disorders, where the role of trauma, abuse, and neglect may be the reason for developing these complex disorders, as psychopathological disorders are difficult to treat (Schmid et al., 2013). Clinical significance of a 
need and utility of a DTD diagnosis continues to be validated in results from research studies, surveys, and other modes of scientific analysis and research.

Child-serving clinicians indicate that a developmental trauma disorder has clinical utility and although there may be overlap with other diagnoses, it is still "discriminable from existing psychiatric diagnoses and their criteria" (Ford, Grasso, Greene, Levine, Spinazzola, \& van der Kolk, 2012, p. 847). 


\section{References}

American Psychiatric Association. (1994). Diagnostic and statistical manual of mental disorders: DSM-IV. Washington, DC: American Psychiatric Association.

American Psychiatric Association, (2017). DSM History, DSM-5. website. Retrieved from: https://www.psychiatry.org/psychiatrists/practice/dsm/history-of-the-dsm

Cloitre, M., Stolbach, B. C., Herman, J. L., Kolk, B. V. D., Pynoos, R., Wang, J., \& Petkova, E. (2009). A developmental approach to complex PTSD: Childhood and adult cumulative trauma as predictors of symptom complexity. Journal of traumatic stress, 22(5), 399-408.

D’Andrea, W., Ford, J., Stolbach, B., Spinazzola, J., \& van der Kolk, B. A. (2012). Understanding interpersonal trauma in children: Why we need a developmentally appropriate trauma diagnosis. American Journal of Orthopsychiatry, 82(2), 187-200.

DeAngelis, T. American Psychiatric Association. (2007, March). 38(3). What A New Diagnosis Would Include. website. Retrieved from: http://www.apa.org/monitor/mar07/include.aspx

Denton, R., Frogley, C., Jackson, S., John, M., \& Querstret, D. (2017). The assessment of developmental trauma in children and adolescents: A systematic review. Clinical child psychology and psychiatry, 22(2), 260-287.

Drabick, D. A., \& Kendall, P. C. (2010). Developmental psychopathology and the diagnosis of mental health problems among youth. Clinical Psychology: Science and Practice, 17(4), 272-280. 
Fang, X., Brown, D. S., Florence, C. S., \& Mercy, J. A. (2012). The economic burden of child maltreatment in the United States and implications for prevention. Child abuse \& neglect, 36(2), 156-165.

Finkelhor, D. (2008). Childhood victimization: Violence, crime, and abuse in the lives of young people. Oxford University Press.

Grossman, F.K., Spinazzola, J., Zucker, M., \& Hopper, E. (2017). Treating adult survivors of childhood emotional abuse and neglect: A new framework. American Journal of Orthopsychiatry, 87(1), 86.

Herman, Judith Lewis. Trauma and Recovery. New York: Basic Books, 1992.

Klasen, F., Oettingen, G., Daniels, J., Post, M., Hoyer, C., \& Adam, H. (2010). Posttraumatic resilience in former Ugandan child soldiers. Child development, 81(4), 1096-1113.

Moretti, M. M., \& Peled, M. (2004). Adolescent-parent attachment: Bonds that support healthy development. Pediatrics \& child health, 9(8), 551-555.

Pearlman, L. A., \& Courtois, C. A. (2005). Clinical applications of the attachment framework: Relational treatment of complex trauma. Journal of traumatic stress, 18(5), 449-459.

Price, M., Spinazzola, J., Musicaro, R., Turner, J., Suvak, M., Emerson, D., \& van der Kolk, B. (2017). Effectiveness of an Extended Yoga Treatment for Women with Chronic Posttraumatic Stress Disorder. The Journal of Alternative and Complementary Medicine, 23(4), 300-309.

Sar, V. (2011). Developmental trauma, complex PTSD, and the current proposal of DSM-5. European Journal of Psychotraumatology, 2(1), 5622. 
Schimmenti, A., \& Caretti, V. (2016). Linking the overwhelming with the unbearable:

Developmental trauma, dissociation, and the disconnected self. Psychoanalytic Psychology, 33(1), 106.

Schmid, M., Petermann, F., \& Fegert, J. M. (2013). Developmental trauma disorder: pros and cons of including formal criteria in the psychiatric diagnostic systems. $B M C$ psychiatry, 13(1), 3.

Stolbach, B. C., Minshew, R., Rompala, V., Dominguez, R. Z., Gazibara, T., \& Finke, R.

(2013). Complex trauma exposure and symptoms in urban traumatized children: A preliminary test of proposed criteria for developmental trauma disorder. Journal of Traumatic Stress, 26(4), 483-491.

U.S. Department of Veterans Affairs. (2016, February 23). PTSD: National Center for PTSD, Complex PTSD. website. Retrieved from: https://www.ptsd.va.gov/professional/ptsd-overview/complex-ptsd.asp

Van der Kolk, B. A. (2002). The assessment and treatment of complex PTSD. Treating trauma survivors with PTSD, 127-156.

Van der Kolk, B. A., Roth, S., Pelcovitz, D., Sunday, S., \& Spinazzola, J. (2005). Disorders of extreme stress: The empirical foundation of a complex adaptation to trauma. Journal of traumatic stress, 18(5), 389-399.

Van der Kolk, B. A. (2005). Developmental trauma disorder: towards a rational diagnosis for children with complex trauma histories. Psychiatric Annals, 35(5) 401-408.

Van der Kolk, B. A., Pynoos, R. S., Cicchetti, D., Cloitre, M., D’Andrea, W., Ford, J. D.,. \& Stolbach, B. C. (2009). Proposal to include a developmental trauma disorder diagnosis for children and adolescents in DSM-V. Unpublished manuscript. 
Van der Kolk, B. A. (2014). The body keeps the score: Brain, mind, and body in the healing of trauma. New York: Viking. 